\title{
Biomechanical Analysis of the Canine Tuberculum Dentale
}

\author{
Ionut Ichim, Michael Swain and Jules Kieser* \\ Department of Oral Sciences, Faculty of Dentistry, University of Otago, Dunedin, New Zealand
}

\begin{abstract}
We evaluate the structural significance of the development of a canine tuberculum dentale by means of three-dimensional finite element analysis. Using a scanned human permanent canine, we construct a computer generated canine, together with alveolar bone and periodontal ligament onto which we morph two cingulum shapes, namely a flat palatal surface and a stylised tuberculum dentale. We then subject the three shapes (flat, normal cingulum, and pronounced
\end{abstract}

The canine tuberculum dentale is a cingular derivative found on the lingual surface of the maxillary anterior teeth (Scott and Turner, 2000). It varies in expression from a low ridge to a well-formed cusplet (Hillson, 1986). While a number of studies have reported on its incidence and expression in modern and archaic populations (e.g., Scott and Dahlberg, 1982; Cucina et al., 1999; Bailey, 2000), to the best of our knowledge none has focused on its biomechanical significance. In this paper, we use three-dimensional finite element analyses on two canines, one with and one without a tuberculum dentale, morphed from a scanned image of a human upper canine, to investigate whether this trait plays a significant role in the structural response of the tooth under functional loading.

\section{MATERIALS and METHODS}

\section{Source model and morphing}

We scanned a human permanent upper canine, extracted for periodontal reasons, on a micro-CT scanner (SkyScan 1072 system). The sections were taken at 15micron intervals, yielding a stack of 1954 slices. Using in-house software, an initial assembly of two meshes of the surfaces and interfaces of the canine was generated. The result was an initial surface mesh that enclosed the volumes of enamel and dentine. The root cementum layer was not modeled because of its particularly small dimensions and the limited relevance for our study.

We then morphed two crown shapes, one with a stylized tuberculum dentale and the other with a flattened palatal surface. Morphing was carried by simultaneously displacing the vertices of the outer and inner surfaces of the enamel volume and keeping the enamel thickness in the morphed models similar to that tuberculum dentale) to a normal occlusal force and we record principal and von Mises stresses in the crowns. Our results show that stresses are concentrated at the cingulum and in the approximal areas, and that these do not differ between the three forms. We conclude that the development of a tuberculum dentale does not confer biomechanical advantage to the human canine. Dental Anthropology 2005;18(2):50-54.

of the original scanned tooth. Hence, three geometrical tooth crowns were generated this way: a source model constructed from the micro-CT data, a model with a stylized tuberculum dentale and model with a flat palatal shape.

\section{Finite element models}

After applying the surface meshing we performed a NURBS conversion (Non-Uniform Rational B-Splines) that defined the respective solid volumes for each of the three models. This was done by patching, using a feature available with a general purpose CAD software (Smurf for Rhinoceros 3D for Windows, Robert McNeel and Associates, USA). Patching consisted of applying quadrilateral NURBS onto the surface of the mesh, hence covering the original mesh with tiles of rational surfaces with tangent continuity that are later joined into closed solids.

Two matching bodies were thus been created, one representing the enamel and the other the dentine, in contact along the entire dentinoenamel junction. For reasons of computational-efficiency, the anatomic roughness of this junction, well captured on the CT reconstruction was neglected and a smoother junction created.

The pulp space was modeled as a void inside the dentine volume, because its Young's modulus is negligibly small compared with that of the surrounding enamel and dentine (Hojjatie and Anusavice, 1990).

${ }^{*}$ Correspondence address: Jules Kieser, Department of Oral Sciences, Faculty of Dentistry, University of Otago, Dunedin, New Zealand

E-mail: jules.kieser@stonebow.otago.ac.nz 
TABLE 1. Material properties used in the present FEA analysis (O'Brien, 2002)

\begin{tabular}{lcc}
\hline Tissue & $\begin{array}{c}\text { Elastic } \\
\text { modulus }\end{array}$ & $\begin{array}{c}\text { Poisson's } \\
\text { ratio }\end{array}$ \\
\hline Enamel & $130 \mathrm{GPa}$ & 0.3 \\
Dentine & $14.7 \mathrm{GPa}$ & 0.3 \\
Periodontal ligament & $12.0 \mathrm{MPa}$ & 0.45 \\
Alveolar bone & $490 \mathrm{MPa}$ & 0.3 \\
\hline
\end{tabular}

We then simulated the periodontal ligament creating a uniform $0.3 \mathrm{~mm}$ shell around the root, and also a bone supporting volume to receive the socket thus created. The upper limit of the bone was set $2 \mathrm{~mm}$ below the cervical line of the tooth, thus simulating the actual anatomic situation (Schroeder, 1991).

A $2.3 \mathrm{~mm}^{2}$ loading area was defined on the crown, circumscribing a hypothetical palatal wear facet where the lower canine occluded. The high-fidelity shape generated from micro-CT allowed an easy recognition of the wear facets and a realistic placement of functional loading was achieved. The loading area was positioned identically in all three models.

The resulting geometric assemblies were imported into general-purpose FEA software (Cosmos DesignStar, Structural Research and Analysis Corp., USA) and meshed using parabolic tetrahedral solid elements. This yielded 62,925 elements for the flat cingulum model, 62,964 elements for the unaltered shape and 63,010 elements for the prominent cingulum model.

The models were rigidly restrained along the lateral and basal surfaces of the bone with the tooth free to move within the defined periodontal space. We assigned isotropic homogenous materials properties for the enamel, dentine and cancellous bone as described in the literature (Table 1). It is known that the tooth structures are made of non-homogenous and anisotropic materials yet the regional property variation is restricted to a microscopic scale and comparisons with real physical specimens have shown that the material behavior is elastic during functions (Kinney et al., 1999; Qin and Swain, 2004).

The periodontal ligament was assumed to be a linear elastic material with an elastic modulus of $12 \mathrm{MPa}$ and Poisson's ratio of 0.45 . We obtained these values in a preliminary analysis by steadily increasing the elastic modulus of the material until the unaltered canine intruded $0.3 \mathrm{~mm}$ under an axial load of $300 \mathrm{~N}$. This mobility was employed to fit previous in vivo mobility data reported in the literature (Muhlemann, 1967). Because this showed that during incision the canine cingulum area was stress-free, we discarded this loading case. Therefore only the palatal contact was prescribed as the loading condition, simulating the occlusion, with the force acting on the previously defined areas.

Because no data were available for the contact angle between the upper and lower canines, we approximated this to be $160^{\circ}$ based on the angle of the incisors (Milot and Stein, 1992). The biting force was estimated at $200 \mathrm{~N}$ (derived from Miyaura et al., 1999) for all three cases. We then assessed the principal stresses $\left(\sigma_{1}\right.$ and $\left.\sigma_{3}\right)$ and von Mises stresses for each loading case.

\section{RESULTS}

For a given loading case, the stress analysis results show that the modest tuberculum dentale shape has little influence if any, upon the structural loading of the canine crown. First principal stress plots reveal two areas of high tension in all three models which are located
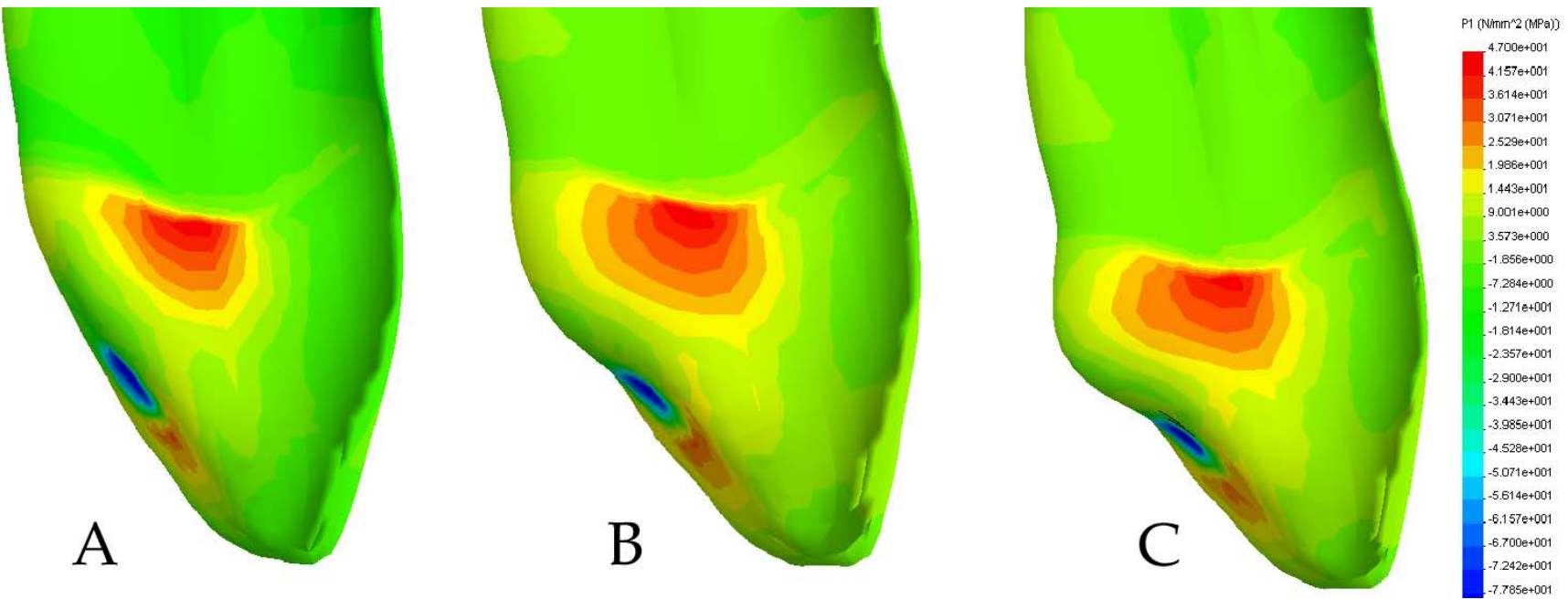

Fig. 1. First principal stress in (A) flat palatal surface, (B) normal, and (C) stylized tuberculum dentale models. Note the constant distribution of tensile stresses on the proximal surfaces of the crown, adjacent to the cementoenamel junction. 

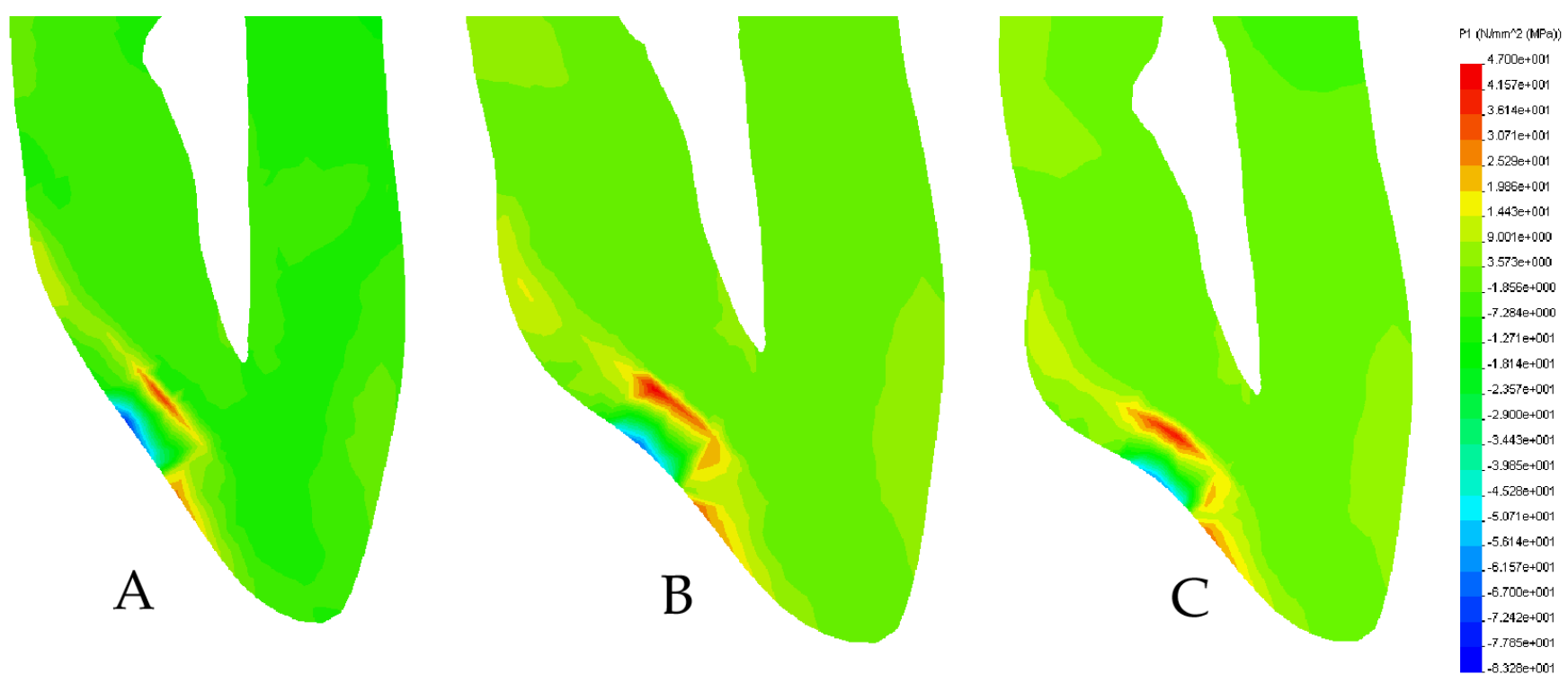

Fig. 2. Axial section plots in (A) flat palatal surface, (B) normal, and (C) stylized tuberculum dentale models.

on the proximal surfaces, close to the cementoenamel junction. The values of this proximal tensile stress are close to the reported ultimate tensile strength of the enamel (Fig. 1).

An axial section shows that tensile stresses follow a similar path in all three canine shapes, with a high of 38-43 $\mathrm{MPa}$ located under the loading area, on the dentinoenamel junction. Tensile loading on the cingulum area increased from $17 \mathrm{MPa}$ in the flat shape to $14 \mathrm{MPa}$ in the normal cingulum and $11 \mathrm{MPa}$ in the tuberculum dentale shape (Fig. 2).
The third principal stress analysis reveals that the compressive stress is located on the buccal half of cervical margin and also at the loading point. The numerical values were similar for all three cases ranging from 47 to $50 \mathrm{MPa}$ (Fig. 3).

Von Mises stresses show a similar pattern in all three models, with two main concentration areas, one along the cervical margin and the other on the loading sites. However, a small decrease in von Mises stresses is recorded parallel with the increase in size of the tuberculum dentale (Fig. 4).
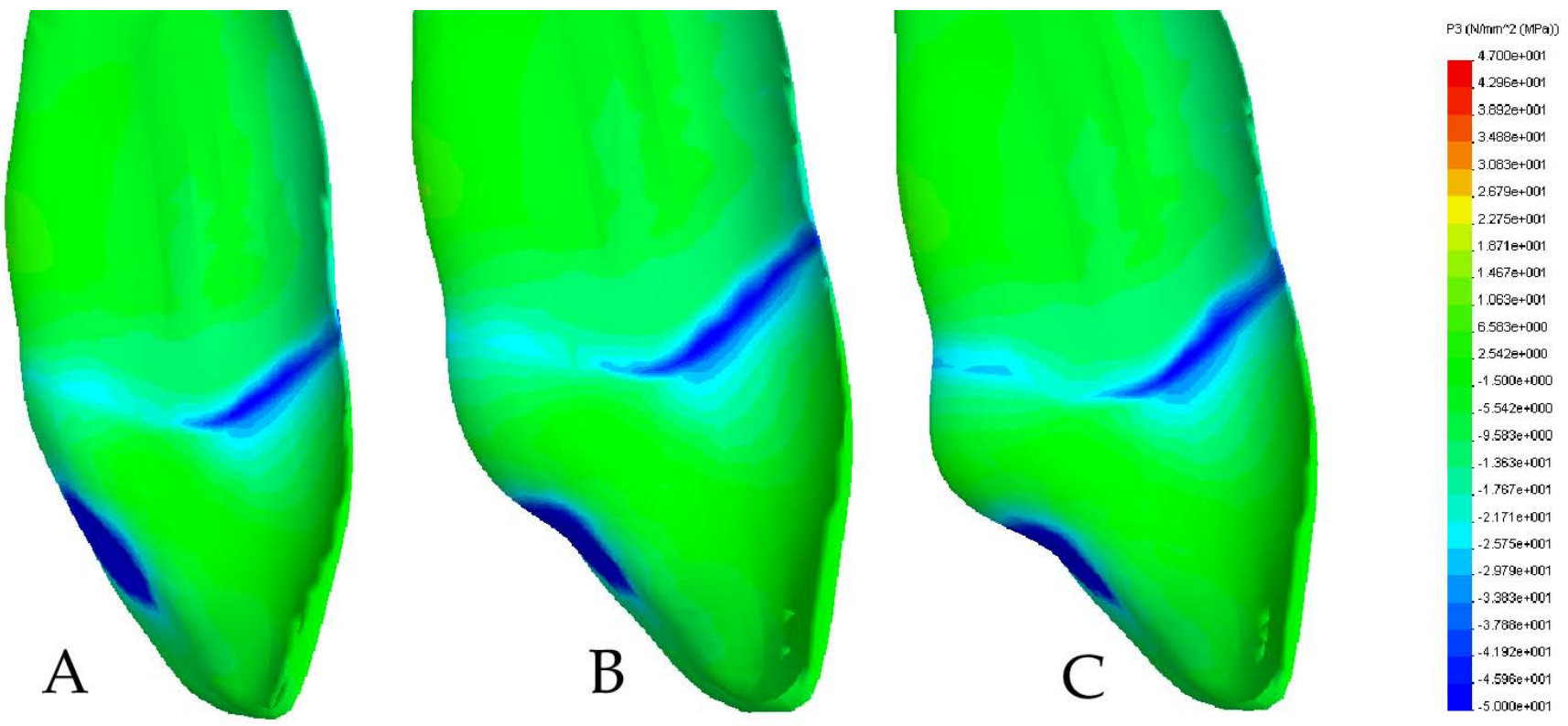

Fig. 3. Axial section plots of the third principal stress in (A) flat palatal surface, (B) normal, and (C) stylized tuberculum dentale models. The distribution of the compressive loading on the buccal half of the CEJ is evident. 

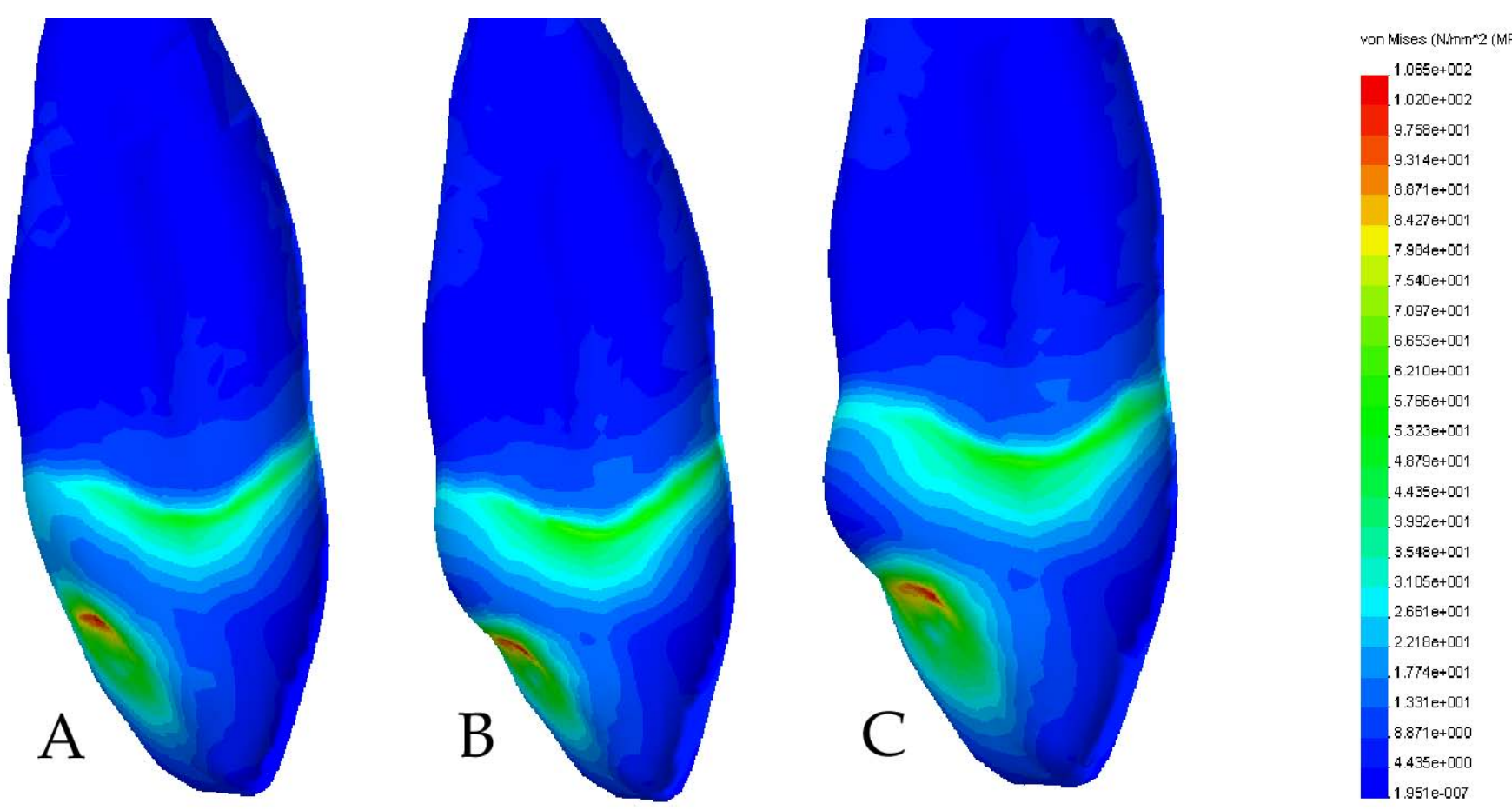

Fig. 4. Von Mises stresses in the (A) flat cingulum, (B) normal, and (C) prominent cingulum models.
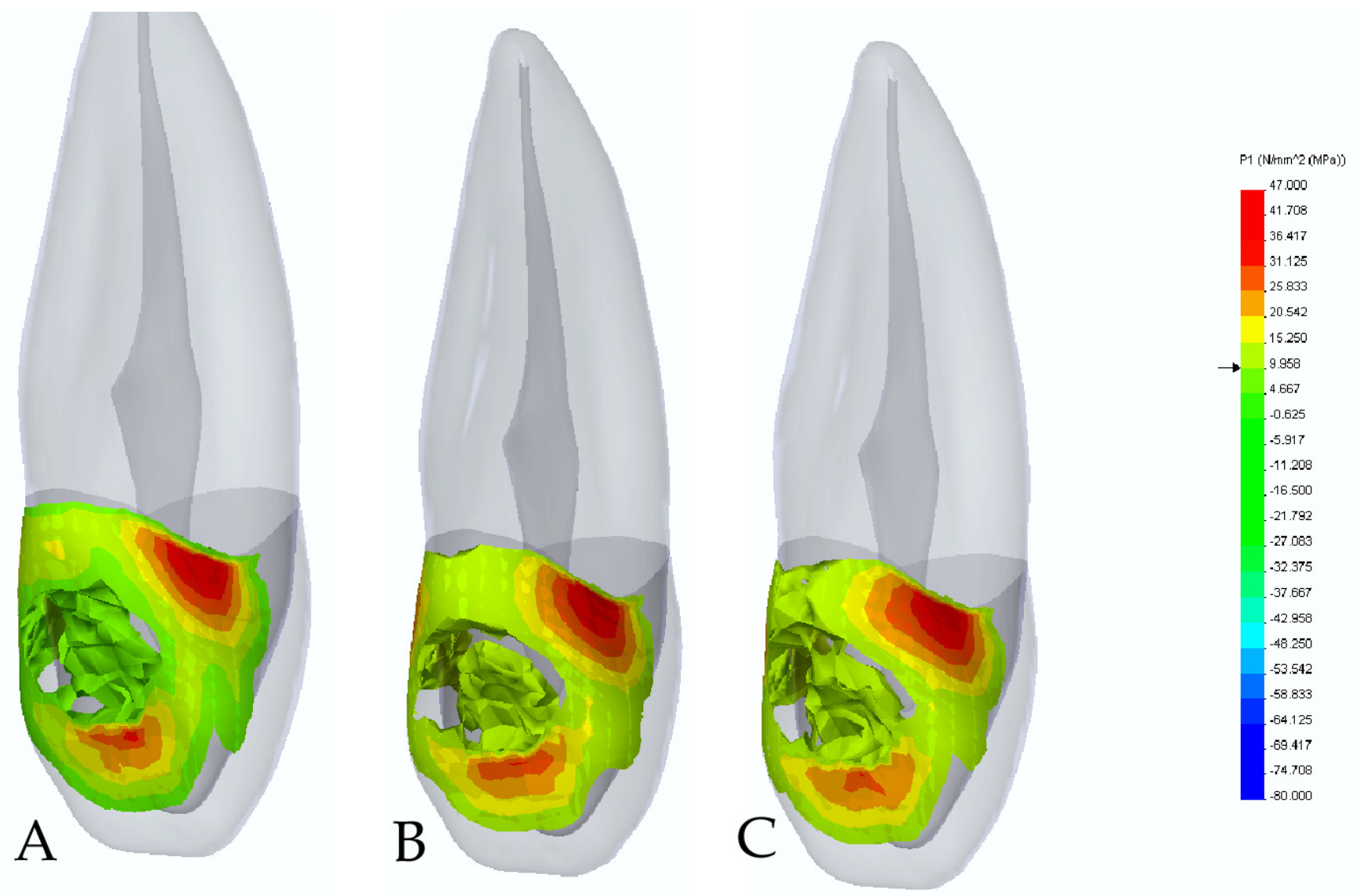

Fig.5. The "resistance frame" inside the crown showing the volume that will experience tensile stresses over 10 MPa. Note the hoop-like shape and the maximal tensions on the proximal surfaces. 


\section{DISCUSSION}

Recently, Bailey (2000) presented data on a number of dental non-metrical traits to ascertain relationships among early and recent human populations. One of her interesting findings is that Neandertals showed an average frequency of $87.5 \%$ for the tuberculum dentale, which contrasts sharply with trait frequencies in British $(25.5 \%)$ and North African (38.8\%) populations. This is not surprising, given the numerous craniodental and postcranial differences in robusticity reported between Neanderthals and modern humans (e.g., Rak, 1986; Stringer and Gamble, 1993; Holliday, 1997). The question now arises, does the tuberculum dentale confer additional robusticity to the typical Neanderthal canine?

In this paper we test the hypothesis that the tuberculum dentale plays a significant role in the structural response of the canine tooth under functional loading. To this end, we compare the numerically determined values of three different shapes of the canine cingulum area under identical loading conditions. For each crown form, we analyze the loading case, the principal stresses, $\sigma_{1}, \sigma_{3}$ and von Mises stresses.

We show that compressive stresses are located mainly on the buccal side, along the cementoenamel junction, with determined values well within the biological material safety limits of enamel (Figs. 1, 2). Tensile and von Mises stresses are dominant on the cervical third of the lingual aspects of the crown in each of the three morphological shapes (Figs 3,4), again with minimal differences between cingulum forms. This strongly suggests that the tuberculum dentale does not in fact strengthen the canine under occlusal loads.

The tensile stress analysis allows for a "resistance frame" to be defined inside the crown which shows the part of the structure that is experiencing the greatest tension (Fig. 5). In structural terms, this frame will provide stiffness to the crown.

The frame thus generated encircles two-thirds of the cervical contour on the lingual aspect of the tooth and extends towards incisal surface, along the marginal ridges of the crown. Neither the locations nor the intensity of the peak tensile stresses are affected by differences in the development of the canine cingulum. Again, this supports the suggestion that the tuberculum dentale does not play a significant role in the structural response of the canine tooth under functional loading.

\section{LITERATURE CITED}

Bailey SE. 2000. Dental morphological affinities among late Pleistocene and recent humans. Dent Anthropology 14:1-8.

Cucina A, Lucci M, Vargiu R, Coppa A. 1999. Dental evidence of biological affinity and environmental conditions in prehistoric Trentino samples from the Neolithic to the Early Bronze Age. Int J Osteoarchaeol 9:404-416.

Hojjatie B, Anusavice KJ. 1990. Three-dimensional finite element analysis of glass-ceramic dental crowns. J Biomech 23:1157-1166.

Hillson S. 1986. Teeth. Cambridge: Cambridge University Press.

Kinney JH, Balooch M, Marshall GW, Marshall SJ. 1999. A micromechanics model of the elastic properties of human dentine. Arch Oral Biol 44:813-822.

Milot, P. Stein RS. 1992. Root fracture in endodontically treated teeth related to post selection and crown design. J Pros Dent 68:428-435.

Miyaura, K, Matsuka Y, Morita M, Yamashita A, Watanabe T. 1999. Comparison of biting forces in different age and sex groups: a study of biting efficiency with mobile and non-mobile teeth. J Oral Rehab 26:223-227.

Muhlemann, HR. 1967. Tooth mobility: a review of clinical aspects and research findings. J Periodontol 38: Suppl: 686-713.

O'Brien, WJ. 1997. Dental materials and their selection. Chicago: Quintessence Publishing.

Qin Q, Swain M. 2004. A micro-mechanics model of dentin mechanical properties. Biomaterials 25:50815090.

Schroeder HE. 1991. Oral structural biology. Zurich: Thieme.

Scott RC, Dahlberg AA. 1982. Microdifferentiation in tooth crown morphology among Indians of the American Southwest. In: Kurtén B, editor. Teeth: form, function and evolution. New York: Columbia University Press, p 259-291.

Scott GR, Turner CG. 2000. The anthropology of modern human teeth. Cambridge: Cambridge University Press. 